cases were $60 \%$ and $25 \%$, respectively. At present, the median survival period after recurrence of operation cases was longer than that of contraindication cases ( 37 months v.s. 13 months ). Our results suggested that one FDG-PET oriented operation roughly corresponded to one year survival benefit with restart.

Conclusion Conclusion: FDG-PET could identify malignant lesions at earlier stage, and was an effective modality to evaluate not only disease spread but distant metastasis for recurrence of colorectal cancer. In this study, we first concretely demonstrated that FDG-PET oriented surgical indication had survival benefit for recurrent colorectal cancer.

Disclosure of Interest None Declared

\section{OC-087 SCREEN-DETECTED COLORECTAL CANCERS ARE ASSOCIATED WITH AN IMPROVED OUTCOME WHEN COMPARED WITH INTERVAL CANCERS WHEN MATCHED FOR STAGE}

doi:10.1136/gutjnl-2013-304907.086

$1,2,3,{ }^{*} \mathrm{M} D$ Gill, $2,3 \mathrm{M}$ Bramble, ${ }^{4} \mathrm{M}$ Hull, ${ }^{1,3} \mathrm{~S}$ Mills, ${ }^{4}$ E Morris, ${ }^{1,3} \mathrm{M}$ Bradburn, ${ }^{5} \mathrm{Y}$ Bury, ${ }^{3,6} \mathrm{C}$ Parker, ${ }^{3} \mathrm{~T}$ Lee, ${ }^{2,3,6} \mathrm{C}$ Rees. ${ }^{1}$ Northern Colorectal Cancer Audit Group, Northumberland; 2University of Durham, Durham; ${ }^{3}$ Northern Region Endoscopy Group, North East UK; ${ }^{4}$ University of Leeds, Leeds; ${ }^{5}$ Royal Victoria Infirmary, Newcastle; ${ }^{6}$ South Tyneside Foundation Trust, South Tyneside, UK

Introduction Colorectal cancers detected through the NHS Bowel Cancer Screening Programme (BCSP) have been shown to have a more favourable outcome compared to non-screen detected cancers. The aim of this study was to identify whether this was solely due to the earlier stage shift of these cancers, or whether there were other factors involved.

Methods A combination of a regional colorectal cancer registry (Northern Colorectal Cancer Audit Group) and the BCSP database were used to identify screen detected cancers and interval cancers (diagnosed after a negative faecal occult blood test, before the next screening round). All cancers were diagnosed between April 2007 and March 2010, within the North East of England. For each Dukes' stage, patient demographics, tumour characteristics, and survival rates were compared between the screen detected and interval cancer groups.

Results 322 screen detected cancers were compared against 192 interval cancers.

Significant differences highlighted in bold, $p<0.05$. Mean follow-up 32 months.

Conclusion With equivalent patient demographics and tumour characteristics, the improved survival of screen detected cancers over interval cancers for Stages $\mathrm{C}$ and $\mathrm{D}$ suggest that there may be a biological difference in the cancers in each group. Although leadtime bias may have a role, this may be related to a tumours propensity to bleed and therefore may reflect detection through current screening tests.

Disclosure of Interest None Declared

\section{OC-088 PROSPECTIVE PILOT STUDY TO INVESTIGATE TRANSCUTANEOUS SACRAL NERVE STIMULATION FOR FAECAL INCONTINENCE}

doi:10.1136/gutjnl-2013-304907.087

1."G Thomas, 'C Norton, 'R J Nicholls, ' $\mathrm{C}$ Vaizey. ${ }^{1}$ St Mark's Hospital and Academic Institute, London, UK

Introduction Sacral nerve stimulation (SNS) is an effective treatment for faecal incontinence (FI). However it is expensive, it requires two operations and has a risk of infection, implant migration and pain. Transcutaneous SNS is non-invasive and cheap. Only one small study has previously reported its use for FI. The aim of this study is to further assess the efficacy of transcutaneous SNS for FI.

Methods Recruited patients self-administered transcutaneous SNS for 12 hours a day, over four weeks. A two week bowel diary was kept for the final two weeks and compared to baseline. St Marks FI scores, a visual analogue scale assessing satisfaction with bowel habit, Rockwood FI QOL scores and SF-36 QOL scores were obtained.

Results Ten patients were recruited. Two achieved complete continence. There were significant reductions in the frequency of FI episodes per week, 9.5 (7.5) to 3 (7.38); $p=0.03$, and in the frequency of defecation per week, 25.5 (19.5) to 14.5 (14.9); $p=0.007$. There was a significant improvement in the ability to defer defecation (1(1.25) to 4.5 (4.5) minutes, $\mathrm{p}=0.02)$. There was a significant improvement in the St Marks FI score, 20 (5.25) to 14.5 (8.0); $p=0.01$. There was a significant improvement in the bowel habit satisfaction visual analogue scale 8.5 (20) to 45 (33); p $=0.008$. There were no significant changes in the Rockwood FI QOL score, or in the SF-36 QOL score. No complications were reported.

Conclusion Transcutaneous SNS appears to be an effective and safe treatment for FI.

Disclosure of Interest None Declared

\section{Pathology free papers}

\section{OC-089 EPIGENETIC CONTROL OF GI INFLAMMATION VIA THE METHYL-BINDING PROTEIN MBD2}

doi:10.1136/gutjnl-2013-304907.088

1.*'G-R Jones, 'P Cook, 'L Webb, 'A MacDonald. 'University of Edinburgh, Edinburgh, UK

Introduction Methyl-CpG binding protein domain protein-2 $(\mathrm{Mbd} 2)$ is a transcriptional co-repressor that binds to methylated DNA. Mbd2 can recruit a nucleosome remodelling complex which contains chromatin remodelling and histone deacetylase properties. Mbd2 deficient mice are viable and fertile. However, they display a dysregulated immune phenotype with an aberrant $\mathrm{T}$ cell cytokine response and susceptibility to intestinal helminth infection (1). This immunological phenotype has not been explored in the GI tract.

Abstract 0C-087 Table

\begin{tabular}{|c|c|c|c|c|c|c|c|c|}
\hline & \multicolumn{2}{|c|}{ Dukes' Stage A } & \multicolumn{2}{|c|}{ Dukes' Stage B } & \multicolumn{2}{|l|}{ Dukes' Stage C } & \multicolumn{2}{|c|}{ Dukes' Stage D } \\
\hline & Screen & Interval & Screen & Interval & Screen & Interval & Screen & Interval \\
\hline Gender & \multicolumn{2}{|c|}{ No Difference } & \multicolumn{2}{|c|}{ No Difference } & More Men & More Women & \multicolumn{2}{|c|}{ No Difference } \\
\hline Deprivation Level & \multicolumn{2}{|c|}{ No Difference } & \multicolumn{2}{|c|}{ No Difference } & \multicolumn{2}{|l|}{ No Difference } & \multicolumn{2}{|c|}{ No Difference } \\
\hline ASA Grade & \multicolumn{2}{|c|}{ No Difference } & \multicolumn{2}{|c|}{ No Difference } & No Difference & & \multicolumn{2}{|c|}{ No Difference } \\
\hline T Stage & \multicolumn{2}{|c|}{ No Difference } & More T3 & More T4 & No Difference & & \multicolumn{2}{|c|}{ No Difference } \\
\hline N Stage & \multicolumn{2}{|l|}{$\mathrm{N} / \mathrm{A}$} & \multicolumn{2}{|l|}{$\mathrm{N} / \mathrm{A}$} & More N1 & More N2 & \multicolumn{2}{|c|}{ No Difference } \\
\hline Tumour Site & \multicolumn{2}{|c|}{ No Difference } & \multicolumn{2}{|c|}{ No Difference } & More Left-Sided & More Right-Sided & \multicolumn{2}{|c|}{ No Difference } \\
\hline Survival Rate & \multicolumn{2}{|c|}{ No Difference } & \multicolumn{2}{|c|}{ No Difference } & Better & Worse & Better & Worse \\
\hline
\end{tabular}

